\title{
Mycoplasma lagogenitalium sp. nov., from the Preputial Smegma of Afghan Pikas (Ochotona rufescens rufescens)
}

\author{
HIDEKI KOBAYASHI, ${ }^{1 *}$ MARTIN RUNGE, ${ }^{2}$ ROSEMARIE SCHMIDT, ${ }^{2}$ MASANORI KUBO, ${ }^{1}$ \\ KOSHI YAMAMOTO, ${ }^{1}$ AND HELGA KIRCHHOFF ${ }^{2}$ \\ National Institute of Animal Health, 3-1-1, Kannondai, Tsukuba, Ibaraki 305, Japan, ${ }^{1}$ and Institute für \\ Mikrobiologie und Tierseuchen, Tierärztliche Hochschule Hannover, D-30173 Hannover, Germany ${ }^{2}$
}

\begin{abstract}
Organisms with characteristics typical of mycoplasmas were isolated from the preputial smegma of Afghan picas (Ochotona rufescens rufescens). The results of growth inhibition tests, metabolic inhibition tests, and immunobinding assays showed that the isolated strains were identical and that they were distinct from previously described Mycoplasma, Entomoplasma, Mesoplasma, and Acholeplasma species. These organisms represent a new species, for which the name Mycoplasma lagogenitalium is proposed. $M$. lagogenitalium ferments glucose, does not hydrolyze arginine or urea, reduces tetrazolium chloride, possesses phosphatase activity, does not digest gelatin or casein, and does not produce films or spots. It lyses sheep erythrocytes and does not adsorb sheep, rabbit, or horse erythrocytes. Cholesterol or serum is required for growth. The growth temperature is $37^{\circ} \mathrm{C}$. The guanine-plus-cytosine content of the DNA is $23.0 \pm 1.0 \mathrm{~mol} \%$. The type strain is M. lagogenitalium 12MS $\left(=\operatorname{ATCC} 7_{00289^{T}}\right)$.
\end{abstract}

Mycoplasmas have been demonstrated in several laboratory animals. They have been isolated from rats and mice $(3,9,14)$, Chinese hamsters $(8)$, guinea pigs $(10)$, and rabbits $(5,11)$, and they often cause severe problems in stock breeding. The Afghan pika (Ochotona rufescens rufescens) is a kind of small rabbit with short ears that belongs to the family Ochotonidae; domestic rabbits (Oryctolagus cuniculus var. domesticus) belong to the family Leporidae. Though the pika is becoming a useful experimental animal for comparative biological research and reproductive and developmental toxicity studies, little is known about the microbial flora of the animal. In the present investigation, 22 apparently healthy animals were examined for mycoplasmas and the isolates were characterized and compared with previously described Mycoplasma, Entomoplasma, Mesoplasma, and Acholeplasma species.

\section{MATERIALS AND METHODS}

Animals. Twelve male and 10 female, apparently healthy, Afghan pikas (Ocho tona rufescens rufescens) were investigated for mycoplasmas by cultivation of swabs taken from eyes, ears, nasal cavity, and urogenital tract. These animals were derived from the same herd in Japan.

Isolation and cultivation of mycoplasmas. Swabs were spread on M-agar containing $21 \mathrm{~g}$ of PPLO broth base (Difco, Detroit, Mich.), $5.0 \mathrm{~g}$ of bacteriological mucin (Difco), $150 \mathrm{ml}$ of heat-inactivated horse serum (Gibco, Grand Island, N.Y.), $25 \mathrm{ml}$ of a $25 \%$ (wt/vol) extract of baker's yeast, $10^{6} \mathrm{IU}$ of penicillin $\mathrm{G}$ potassium (Banyu Co. Ltd., Tokyo, Japan), $830 \mathrm{ml}$ of distilled water, and $12 \mathrm{~g}$ of Noble agar (Difco). After that the swabs were suspended in $2 \mathrm{ml}$ of M-broth (the same composition as $\mathbf{M}$-agar, but without agar). Subcultivation from Mbroth to $\mathrm{M}$-agar was done after incubation for 2 or 3 days. Agar plates and broth cultures were incubated at $37^{\circ} \mathrm{C}$ under aerobic and anaerobic conditions for up to 4 weeks. Isolated mycoplasma strains were filter cloned five times with 450 nm-pore-size filters (19).

Morphological studies. The mycoplasma colonies were examined with a stereomicroscope. The cellular morphology of the organisms was assessed by darkfield microscopy. Ultrathin sections of organisms were prepared as described previously $(20)$.

Filtration studies. Cultures (after $48 \mathrm{~h}$ of incubation) were filtered through membrane filters (Sartorius, Göttingen, Germany) with pore diameters of 200 and $450 \mathrm{~nm}$. The numbers of CFU per milliliter in the filtrates were determined by plating the filtrates onto agar and were compared with the CFU per milliliter in an unfiltered culture dilution.

Reversion experiments. The organisms were subcultured five times with liquid or solid medium lacking penicillin or any other antimicrobial agent and were

* Corresponding author. Phone: (81) 298-38-7739. Fax: (81) 298-387740. E-mail: reptile@niah.affrc.go.jp. incubated at $37^{\circ} \mathrm{C}$. After 4 days of incubation, agar plates were examined for alterations in the morphology of the colonies.

Sterol requirement. The sterol requirement was determined by a standard liquid culture technique $(6,7)$ using Mycoplasma caviae $\mathrm{G}^{2} 22^{\mathrm{T}}$ and Acholeplasma laidlawii $\mathrm{PG} 8^{\mathrm{T}}$ as controls.

Biochemical test. Isolated mycoplasmas were examined for metabolism of glucose, hydrolysis of arginine and urea, production of films and spots, phosphatase activity, reduction of tetrazolium chloride, and digestion of casein and gelatin $(1,2,21)$. Hemolysis was tested by the overlay technique (1) using sheep erythrocytes. Hemabsorption was investigated with sheep, rabbit, and horse erythrocytes according to the method described by Sobeslavsky et al. (16).

DNA base composition. The guanine-plus-cytosine $(\mathrm{G}+\mathrm{C})$ content of the DNA of strain $12 \mathrm{MS}$ was determined with the thermal denaturation temperature (13). DNAs extracted from Escherichia coli and Mycoplasma arthritidis, with known $\mathrm{G}+\mathrm{C}$ contents, were included as controls.

Serological studies. Antisera were prepared by the method described by Morton and Roberts (15). A serological comparison of strain $12 \mathrm{MS}^{\mathrm{T}}$ with the other strains isolated from Afghan pikas and with members of previously described Mollicutes species was performed with growth inhibition tests (4), metabolism inhibition tests (18), and immunobinding assays with colonies transferred to nitrocellulose (12). Tests with strain $12 \mathrm{MS}^{\mathrm{T}}$ were done in two directions; i.e. reference antisera were tested with strain $12 \mathrm{MS}^{\mathrm{T}}$ and antiserum against strain $12 \mathrm{MS}^{\mathrm{T}}$ was tested with type strains of previously described mollicutes. The antisera and type strains used for the serological investigations are listed in Table 1.

\section{RESULTS AND DISCUSSION}

Isolation of mycoplasmas. Mycoplasmas were isolated from the preputial smegma from 7 of the 12 male Afghan pikas investigated and were described as strains 1LL, 5MM, 8MS, $12 \mathrm{MS}^{\mathrm{T}}, 14 \mathrm{SS}, 16 \mathrm{LM}$, and $21 \mathrm{MS}$. All seven strains grew on the agar plates directly inoculated with the swabs under both aerobic and anaerobic conditions. No mycoplasmas could be isolated from the eyes, ears, nasal cavity, or mouth of these animals or 15 other animals investigated after enrichment in M-broth or by direct inoculation of agar plates, nor could mycoplasmas be demonstrated in the urogenital tract of the 10 female Afghan pikas. After cloning procedures, 21 clones (three from each of the seven isolates) were selected and used for the biochemical and serological investigations. A clone from strain 12MS was chosen as the type strain.

Morphology and ultrastructure. The colonies of strain $12 \mathrm{MS}^{\mathrm{T}}$ and of other mycoplasma strains isolated from Afghan pikas had a typical fried-egg appearance (Fig. 1A). Ultrathin sections revealed the cell membrane and the absence of a cell wall (Fig. 1B). The cells of strain $12 \mathrm{MS}^{\mathrm{T}}$ appeared to be mainly 
TABLE 1. Mycoplasma, Entomoplasma, Mesoplasma, and Acholeplasma strains and antisera used in comparative serological tests with the mycoplasmas from Afghan pikas

\begin{tabular}{|c|c|c|}
\hline \multirow{2}{*}{ Strain(s) } & \multicolumn{2}{|c|}{ Source $(\mathrm{s})^{a}$} \\
\hline & Mollicutes & Antisera \\
\hline Mycoplasma adleri $\mathrm{G}-145^{\mathrm{T}}$ & FCR & FCR \\
\hline M. agalactiae $\mathrm{PG}^{\mathrm{T}}$ & IRC & IRC, NIAH \\
\hline M. alkalescens $\mathrm{D} 12^{\mathrm{T}}$ & NIH & NIH, NIAH \\
\hline M. alvi Ilsley $^{\mathrm{T}}$ & IRC & IRC \\
\hline M. anatis $1340^{\mathrm{T}}$ & NIH & NIH, IMT, NIAH \\
\hline M. anseris $1219^{\mathrm{T}}$ & VMR & VMR \\
\hline M. arginini $\mathrm{G} 230^{\mathrm{T}}$ & $\mathrm{NIH}$ & NIH, IMT, NIAH \\
\hline M. arthritidis PG6 ${ }^{\mathrm{T}}$ & NIH & NIH, IMT, NIAH \\
\hline M. auris $\mathrm{U}_{1 \mathrm{~A}^{\mathrm{T}}}$ & FCR & FCR \\
\hline M. bovigenitalium $\mathrm{PG} 11^{\mathrm{T}}$ & $\mathrm{NIH}$ & NIH, IMT, NIAH \\
\hline M. bovirhinis $\mathrm{PG} 43^{\mathrm{T}}$ & $\mathrm{NIH}$ & NIH, IMT, NIAH \\
\hline M. bovis Donetta ${ }^{\mathrm{T}}, \mathrm{PG} 45^{\mathrm{T}}$ & IRC & IRC, IMT, NIAH \\
\hline M. bovoculi $\mathrm{M} 165 / 69^{\mathrm{T}}$ & IRC & IRC, NIAH \\
\hline M. buccale $\mathrm{CH} 20247^{\mathrm{T}}$ & NIH & NIH, NIAH \\
\hline M. buteonis $\mathrm{Bb} / \mathrm{T}^{2} \mathrm{~g}^{\mathrm{T}}$ & IMT & IMT \\
\hline M. californicum $\mathrm{ST}^{\mathrm{T}}$ & IRC & IRC, NIAH \\
\hline M. canadense $275 \mathrm{C}^{\mathrm{T}}$ & IRC & IRC, IMT \\
\hline M. canis $\mathrm{PG} 14^{\mathrm{T}}$ & NIH & NIH, IMT, NIAH \\
\hline $\begin{array}{l}\text { M. capricolum subsp. capri- } \\
\text { colum California Kid }\end{array}$ & IRC & IRC, NIAH \\
\hline $\begin{array}{l}\text { M. capricolum subsp. capri- } \\
\text { pneumoniae } \mathrm{F} 38^{\mathrm{T}}\end{array}$ & IRC & NIH, IMT \\
\hline M. caviae $\mathrm{G} 122^{\mathrm{T}}$ & IRC & NIH, IMT, NIAH \\
\hline M. cavipharyngis $117 \mathrm{C}^{\mathrm{T}}$ & FCR & FCR, NIAH \\
\hline M. citelli $\mathrm{RG}-2 \mathrm{C}^{\mathbf{T}}$ & IRC & IRC, NIAH \\
\hline $\begin{array}{l}\text { M. cloacale } 383^{\mathrm{T}} \text {, } \\
\text { ATCC } 35276^{\mathrm{T}}\end{array}$ & FCR, ATCC & FCR, NIAH \\
\hline M. collis $58 \mathrm{~B}^{\mathrm{T}}$ & FCR & FCR, NIAH \\
\hline M. columbinasale $694^{\mathrm{T}}$ & IRC & IRC \\
\hline M. columbinum MMP-1 ${ }^{\mathrm{T}}$ & IRC & IRC \\
\hline M. columborale $\mathrm{MMP}-4^{\mathrm{T}}$ & IRC & IRC \\
\hline M. conjunctivae HRC581 ${ }^{\mathrm{T}}$ & IRC & NIH, IMT, NIAH \\
\hline M. corogypsi $\mathrm{BV}^{\mathrm{T}}$ & FCR & IMT, FCR \\
\hline M. cottewii $\mathrm{VIS}^{\mathrm{T}}$ & FCR & FCR \\
\hline M. cricetuli $\mathrm{CH}^{\mathrm{T}}$ & FCR & FCR, NIAH \\
\hline M. cynos $\mathrm{H} 831^{\mathrm{T}}$ & IRC & IRC, NIAH \\
\hline M. crocodyli MP145 & IMT & IMT \\
\hline M. dispar $462 / 2^{\mathrm{T}}$ & IRC & IRC, IMT, NIAH \\
\hline M. edwardii $\mathrm{PG} 24^{\mathrm{T}}$ & IRC & IRC, IMT, NIAH \\
\hline M. elephantis $\mathrm{E} 42^{\mathrm{T}}$ & MRC, FCR & IMT \\
\hline M. equigenitalium $\mathrm{T} 37^{\mathrm{T}}$ & IMT & IMT, NIAH \\
\hline M. equirhinis $\mathrm{M} 432 / 72^{\mathrm{T}}$ & LIRA & LIRA, IMT, NIAH \\
\hline M. falconis $\mathrm{H} / \mathrm{T}^{\mathrm{T}}$ & IMT & IMT \\
\hline $\begin{array}{l}\text { M. fastidiosum } 4822^{\mathrm{T}} \text {, } \\
\text { ATCC } 33229^{\mathrm{T}}\end{array}$ & IRC, ATCC & IRC, NIAH \\
\hline $\begin{array}{l}\text { M. faucium DC333 } \\
{\text { ATCC } 25293^{\mathrm{T}}}^{\mathrm{T}} \text {, }\end{array}$ & NIH, ATCC & NIH, NIAH \\
\hline $\begin{array}{l}\text { M. felifaucium } \mathrm{PU}^{\mathrm{T}} \text {, } \\
\text { ATCC } 43428^{\mathrm{T}}\end{array}$ & MRC, ATCC & MRC, NIAH \\
\hline $\begin{array}{l}\text { M. feliminutum Ben }{ }^{\mathrm{T}} \text {, } \\
\text { ATCC } 25749^{\mathrm{T}}\end{array}$ & IRC, ATCC & IRC, NIAH \\
\hline M. felis $\mathrm{CO}^{\mathrm{T}}$ & IRC & NIH, IMT, NIAH \\
\hline M. fermentans $\mathrm{PG} 18^{\mathrm{T}}$ & $\mathrm{NIH}$ & NIH, IMT, NIAH \\
\hline $\begin{array}{l}\text { M. flocculare Ms } 42^{\mathrm{T}} \text {, } \\
\text { ATCC } 43428^{\mathrm{T}}\end{array}$ & SVS, ATCC & SVS, NIAH \\
\hline M. gallinaceum $\mathrm{DD}^{\mathrm{T}}$ & NIH & NIH, IMT \\
\hline M. gallinarum $\mathrm{PG}^{\mathrm{T}} \mathrm{T}^{\mathrm{T}}$ & NIH & NIH \\
\hline M. gallisepticum PG31 & NIH & NIH, IMT, NIAH \\
\hline M. gallopavonis $\mathrm{WR} 1^{\mathrm{T}}$ & IRC & IRC \\
\hline M. gateae $\mathrm{CS}^{\mathrm{T}}$ & IRC & IRC, IMT, NIAH \\
\hline M. genitalium $\mathrm{G} 37^{\mathrm{T}}$ & FCR & FCR, NIAH \\
\hline $\begin{array}{l}\text { M. glycophilum } 486^{\mathrm{T}} \text {, } \\
\text { ATCC } 35277^{\mathrm{T}}\end{array}$ & FCR, ATCC & FCR, NIAH \\
\hline M. gypis $\mathrm{B} 1 / \mathrm{T} 1^{\mathrm{T}}$ & IMT & IMT \\
\hline M. hominis PG21 $1^{\mathrm{T}}$ & NIH & NIH, NIAH \\
\hline M. hyopharyngis $\mathrm{H} 3-6 \mathrm{BF}^{\mathrm{T}}$ & FCR & FCR, NIAH \\
\hline M. hyopneumoniae $\mathrm{J}^{\mathrm{T}}$ & SVS & SVS, IMT, NIAH \\
\hline M. hyorhinis $\mathrm{BTS}^{\mathrm{T}}$ & NIH & NIH, CVM, NIAH \\
\hline
\end{tabular}

TABLE $1-$ Continued

\begin{tabular}{|c|c|c|}
\hline \multirow{2}{*}{ Strain(s) } & \multicolumn{2}{|c|}{ Source $(\mathrm{s})^{a}$} \\
\hline & Mollicutes & Antisera \\
\hline M. hyosynoviae $\mathrm{S} 16^{\mathrm{T}}$ & CVM & CVM, IMT, NIAH \\
\hline M. imitans $4229^{\mathrm{T}}$ & FCR & FCR \\
\hline M. indiense $3 \mathrm{~T}^{\mathrm{T}}$ & FCR & FCR \\
\hline M. iners $\mathrm{PG} 30^{\mathrm{T}}$ & $\mathrm{NIH}$ & NIH \\
\hline M. iowae $695^{\mathrm{T}}$ & IRC & IRC \\
\hline M. leocaptivus $3 \mathrm{~L} 2^{\mathrm{T}}$ & NCTC, FCR & NCTC, FCR \\
\hline M. leopharyngis LL2 ${ }^{\mathrm{T}}$ & NCTC, FCR & NCTC, FCR \\
\hline $\begin{array}{l}\text { M. lipofaciens R171 }{ }^{\mathrm{T}} \text {, } \\
\text { ATCC } 35015^{\mathrm{T}}\end{array}$ & FCR, ATCC & FCR, NIAH \\
\hline M. lipophilum Maby $^{\mathrm{T}}$ & NIH & NIH, NIAH \\
\hline M. maculosum $\mathrm{PG} 15^{\mathrm{T}}$ & $\mathrm{NIH}$ & NIH, NIAH \\
\hline M. meleagridis $17529^{\mathrm{T}}$ & IRC & IRC, NIAH \\
\hline M. moatsii MK405 $5^{\mathrm{T}}$ & IRC & IRC, IMT, NIAH \\
\hline M. mobile $163 \mathrm{~K}^{\mathrm{T}}$ & IMT & IMT \\
\hline M. molare $\mathrm{H} 542^{\mathrm{T}}$ & IRC & IRC, IMT, NIAH \\
\hline $\begin{array}{l}\text { M. muris RIII4 } 4^{\mathrm{T}} \text {, } \\
\text { ATCC } 33757^{\mathrm{T}}\end{array}$ & FCR, ATCC & FCR, NIAH \\
\hline M. mustelae $\mathrm{MX}^{\mathrm{T}}$ & FCR & FCR, NIAH \\
\hline $\begin{array}{l}\text { M. mycoides subsp. } \\
\text { mycoides } \text { PG1 }^{\mathrm{T}}\end{array}$ & IRC & IRC, NIAH \\
\hline $\begin{array}{l}\text { M. mycoides subsp. } \\
\text { capri } \text { PG3 }^{\mathrm{T}}\end{array}$ & IRC & IRC, NIAH \\
\hline M. neurolyticum Type $\mathrm{A}^{\mathrm{T}}$ & NIH & NIH, IMT, NIAH \\
\hline M. opalescens $\mathrm{MH} 5408^{\mathrm{T}}$ & IRC & IRC, IMT, NIAH \\
\hline M. orale $\mathrm{CH} 19299^{\mathrm{T}}$ & NIH & NIH, NIAH \\
\hline M. ovipneumoniae $\mathrm{Y} 98^{\mathrm{T}}$ & IRC & IRC, IMT, NIAH \\
\hline M. oxoniensis $128^{\mathrm{T}}$ & FCR & FCR \\
\hline M. penetrans GTU $54^{\mathrm{T}}$ & FCR & FCR \\
\hline M. phocacerebrale $1049^{\mathrm{T}}$ & IMT & IMT \\
\hline M. phocarhinis $852^{\mathrm{T}}$ & IMT & IMT \\
\hline M. phocidae $105^{\mathrm{T}}$ & MAFC & MAFC \\
\hline $\begin{array}{l}\text { M. pirum HRC } 70-159^{\mathrm{T}} \text {, } \\
\text { ATCC } 25960^{\mathrm{T}}\end{array}$ & FCR, ATCC & FCR, NIAH \\
\hline M. pneumoniae $\mathbf{F H}^{\mathrm{T}}$ & NIH & NIH, IMT \\
\hline M. primatum $\mathrm{HRC} 292^{\mathrm{T}}$ & NIH & NIH \\
\hline M. pullorum $\mathrm{CKK}^{\mathrm{T}}$ & IRC & IRC \\
\hline M. pulmonis $\mathrm{PG} 34^{\mathrm{T}}, \mathrm{Ash}^{\mathrm{T}}$ & NIH & NIH, IMT, NIAH \\
\hline M. putrefaciens $\mathrm{KS}-1^{\mathrm{T}}$ & IRC & IRC, IMT, NIAH \\
\hline M. salivarium $\mathrm{PG} 20^{\mathrm{T}}$ & NIH & NIH, NIAH \\
\hline M. simbae $\mathrm{LX}^{\mathrm{T}}$ & NCTC, FCR & NCTC, FCR \\
\hline M. spermatophilum AH $159^{\mathrm{T}}$ & FCR & FCR \\
\hline M. spumans $\mathrm{PG} 13^{\mathrm{T}}$ & $\mathrm{NIH}$ & NIH, IMT, NIAH \\
\hline $\begin{array}{l}\text { M. sualvi Mayfield } \mathrm{B}^{\mathrm{T}} \text {, } \\
\text { ATCC } 33004^{\mathrm{T}}\end{array}$ & GIRA, ATCC & GIRA, NIAH \\
\hline M. subdolum $\mathrm{TB}^{\mathrm{T}}$ & IMT & IMT, NIAH \\
\hline M. synoviae WVU $1853^{\mathrm{T}}$ & IRC & IRC, NIAH \\
\hline $\begin{array}{l}\text { M. testudinis } 01008^{\mathrm{T}} \text {, } \\
\text { ATCC } 43263^{\mathrm{T}}\end{array}$ & MRC, ATCC & MRC, NIAH \\
\hline M. verecundum $107^{\mathrm{T}}$ & IRC & IRC, IMT, NIAH \\
\hline M. yeatsii $\mathrm{GIH}^{\mathrm{T}}$ & FCR & FCR \\
\hline $\begin{array}{l}\text { Entomoplasma ellychniae } \\
\text { ELCN }^{\mathrm{T}}\end{array}$ & FCR & FCR \\
\hline E. lucivorax $\mathrm{PIPN}-2^{\mathrm{T}}$ & FCR & FCR \\
\hline E. luminosum $\mathrm{PIMN}^{\mathrm{T}}$ & FCR & FCR \\
\hline E. melaleucae $\mathrm{M}^{\mathrm{T}}$ & FCR & FCR \\
\hline E. somnilux PYAN-1 ${ }^{\mathrm{T}}$ & FCR & FCR \\
\hline $\begin{array}{l}\text { Mesoplasma entomophilum } \\
\text { TAC }^{\mathbf{T}}\end{array}$ & FCR & FCR \\
\hline M. florum $\mathrm{L}^{\mathrm{T}}$ & FCR & FCR \\
\hline M. lactucae $831-\mathrm{C} 4^{\mathrm{T}}$ & FCR & FCR \\
\hline M. seiffertii $\mathrm{F} 7^{\mathrm{T}}$ & FCR & FCR \\
\hline $\begin{array}{l}\text { Acholeplasma axanthum } \\
\text { S- } 743^{\mathrm{T}}\end{array}$ & IRC & IRC, IMT \\
\hline A. cavigenitalium $\mathrm{GP}^{\mathrm{T}}$ & FCR & FCR \\
\hline A. equifetale $\mathrm{C} 112^{\mathrm{T}}$ & IMT & IMT \\
\hline A. granularum BTS $39^{\mathrm{T}}$ & NIH & NIH, IMT \\
\hline A. hippikon $\mathrm{C} 1^{\mathrm{T}}$ & IMT & IMT \\
\hline A. laidlawii $\mathrm{PG}^{\mathrm{T}}$ & $\mathrm{NIH}$ & NIH, IMT \\
\hline A. modicum $\mathrm{PG} 49^{\mathrm{T}}$ & IRC & IRC, IMT \\
\hline
\end{tabular}


TABLE $1-$ Continued

\begin{tabular}{lll}
\hline \multicolumn{1}{c}{ Strain(s) } & \multicolumn{2}{c}{ Source(s) $^{a}$} \\
\cline { 2 - 3 } & \multicolumn{1}{c}{ Mollicutes } & \multicolumn{1}{c}{ Antisera } \\
\hline A. morum $72-43^{\mathrm{T}}$ & FCR & FCR \\
A. multilocale $\mathrm{PN} 525^{\mathrm{T}}$ & MRC & MRC \\
A. oculi $19 \mathrm{~L}^{\mathrm{T}}$ & IRC & IRC, IMT \\
A. parvum $\mathrm{H}^{\mathrm{T}} 23 \mathrm{M}^{\mathrm{T}}$ & FCR, VFV & FCR, VFV \\
Bovine serogroup & IRC & IRC \\
7 PG50 & & \\
\hline
\end{tabular}

a Abbreviations: ATCC, American Type Culture Collection, Rockville, Md.; CVM, R. F. Ross, College of Veterinary Medicine, lowa State University, Ames; FCR, J. G. Tully, Mycoplasma Section, Frederick Cancer Research on Animal Disease, Compton, Newbury, Berkshire, England; IMT, H. Kirchhoff, Institut für Mikrobiologie und Tierärztliche Hochschule Hannover, Hannover, Germany; IRC, E. A. Freundt, Food and Agriculture Organization, World Health Organization, International Reference Centre for Animal Mycoplasmas, Aarhus, Denmark; LIRA, R. Lemcke, Institute for Research of Animal Diseases, Compton, Newbury, Berkshire, England; MAFC, H. L. Ruhnke, Ministry of Agriculture and Food, Veterinary Laboratory Services, Guelph, Ontario, Canada; MRC, A. Hill, Medical Research Council Laboratories, Carshalton, Surrey, England; NCTC, National Collection of Type Cultures, London, England; NIAH, K. Yamamoto and $\mathbf{H}$. Kobayashi, National Institute of Animal Health, Tsukuba, Ibaraki, Japan; NIH, M. F. Barile and J. G. Tully, National Institutes of Health, Bethesda, Md.; SVS, N. F. Friis, Statens Veterinaere Serum Laboratorium, Copenhagen, Denmark; VFV, M. Ogata, Department of Veterinary Public Health, Azabu University, Fuchinobe Sagamihara, Kanagawa, Japan; VMR, L. Stipkovits, Veterinary Medical Research Institute, Hungarian Academy of Science, Budapest, Hungary.

coccoid. No motility was observed when living strain $12 \mathrm{MS}^{\mathrm{T}}$ cells were examined by dark-field microscopy.

Filtration characteristics. Filtration of a broth culture of strain $12 \mathrm{MS}^{\mathrm{T}}$ reduced the viable count from $2.3 \times 10^{8} \mathrm{CFU} / \mathrm{ml}$ in the original culture to $5.5 \times 10^{7} \mathrm{CFU} / \mathrm{ml}$ in the $450-\mathrm{nm}$ pore-size membrane filtrate and to $2.3 \times 10^{5} \mathrm{CFU} / \mathrm{ml}$ in the 200-nm-pore-size membrane filtrate.

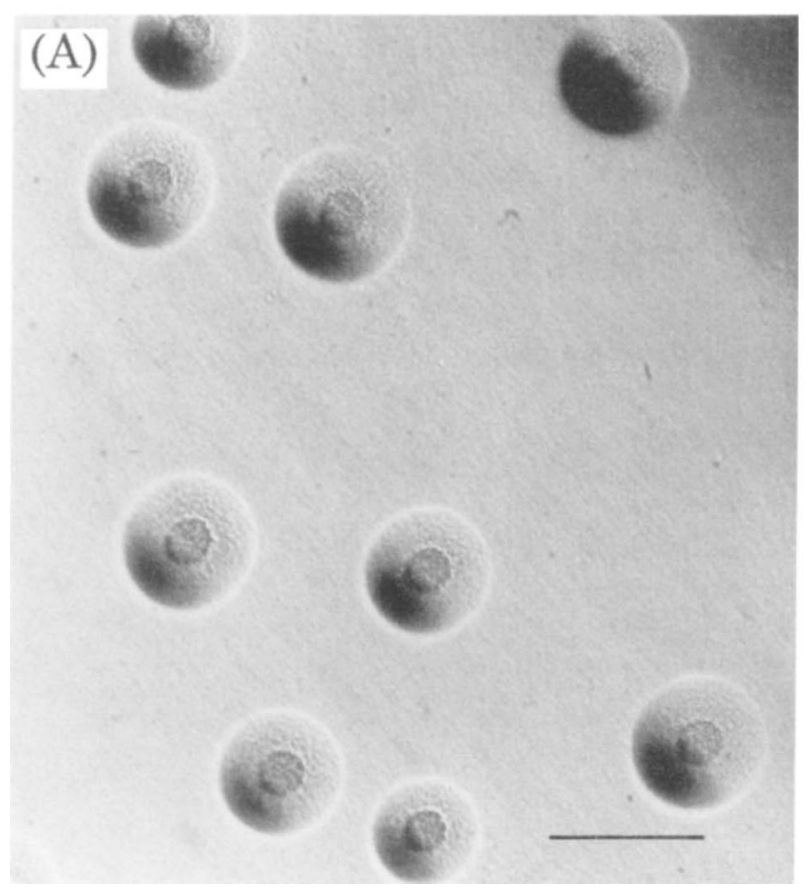

Reversion studies. Strain $12 \mathrm{MS}^{\mathbf{T}}$ and several other mycoplasma strains from Afghan pikas were tested for reversion to bacterial forms. No reversion was observed after five consecutive passages in media without penicillin or other antimicrobial agents.

Sterol requirement. Strain $12 \mathrm{MS}^{\mathrm{T}}$ required cholesterol for growth. The organisms could not be cultured in medium without cholesterol. There were, however, progressively increasing levels of growth when cholesterol was added at concentrations of $1,5,10$ and $20 \mu \mathrm{g} / \mathrm{ml}$ to the medium (Table 2).

Biochemical characteristics. The biochemical activities of strain $12 \mathrm{MS}^{\mathrm{T}}$ and the other strains investigated were identical. They all fermented glucose, reduced tetrazolium chloride, and possessed phosphatase activity. They did not hydrolyze arginine or urea, did not digest gelatin or casein, and did not produce films or spots. They lysed sheep erythrocytes but did not adsorb sheep, rabbit, or horse erythrocytes.

DNA base composition. The $\mathrm{G}+\mathrm{C}$ content of the DNA of strain $12 \mathrm{MS}^{\mathrm{T}}$ was $23 \pm 1.0 \mathrm{~mol} \%$.

Serological characteristics. The 21 mycoplasma strains from Afghan pikas reacted identically in the growth inhibition and metabolic inhibition tests, indicating that they belong to the same species. In immunobinding assays we observed in some of the repeated investigations weak reactions between strain $12 \mathrm{MS}^{\mathrm{T}}$ and other Mycoplasma spp., such as $M$. caviae $\mathrm{G} 122^{\mathrm{T}}$, M. cricetuli $\mathrm{CH}^{\mathrm{T}}, M$. edwardii $\mathrm{PG} 24^{\mathrm{T}}, M$. equigenitalium $\mathrm{T} 37^{\mathrm{T}}$, $M$. equirhinis $\mathrm{M} 432 / 72^{\mathrm{T}}, M$. felifaucium $\mathrm{PU}^{\mathrm{T}}, M$. felis $\mathrm{CO}^{\mathrm{T}}$, M. glycophilum $486^{\mathrm{T}}, M$. hyosynoviae $\mathrm{S} 16^{\mathrm{T}}$, and $M$. meleagridis $17529^{\mathrm{T}}$. These reactions did not appear in the growth and metabolism inhibition tests. No serological reactions were observed in immunobinding assays, growth inhibition tests, or metabolic inhibition tests between strain $12 \mathrm{MS}^{\mathrm{T}}$ and other Mycoplasma, Entomoplasma, Mesoplasma, and Acholeplasma species listed in Table 1.

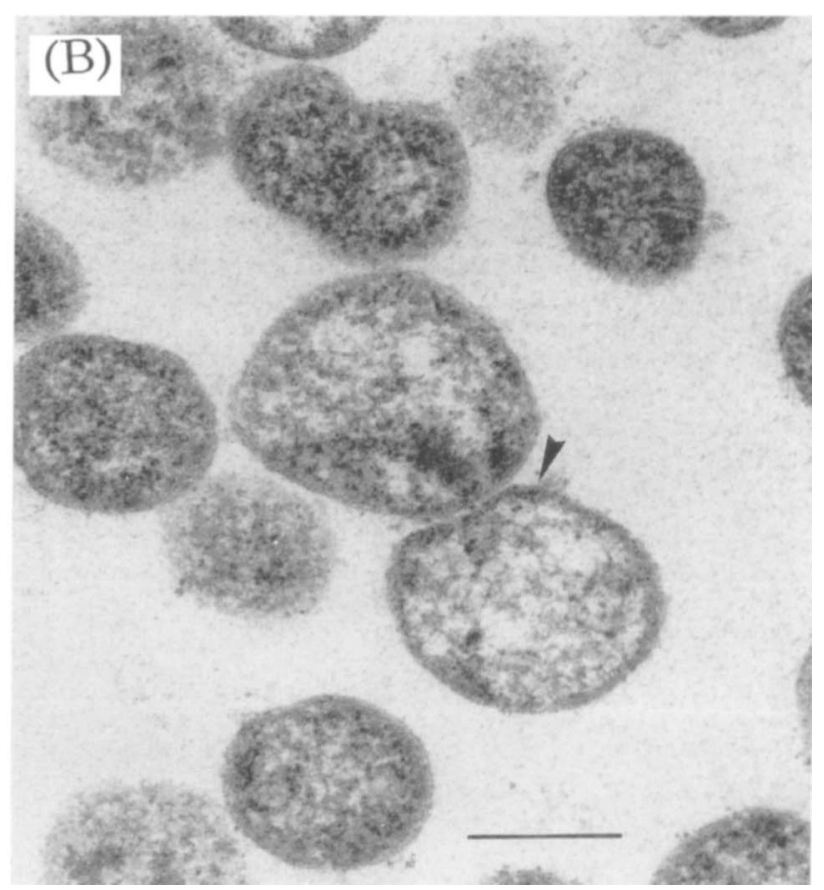

FIG. 1. (A) Typical fried-egg morphology of colonies of strain $12 \mathrm{MS}^{\mathrm{T}}$ grown on agar medium after incubation under aerobic conditions for 2 days. Bar $=1 \mathrm{~mm}$. (B) Electron micrograph of an ultrathin section of strain $12 \mathrm{MS}^{\mathrm{T}}$ cells, showing the absence of a cell wall and the presence of a cell membrane (arrow). The section was stained with $2 \%$ aqueous uranyl acetate and Raymond lead citrate. Bar $=200 \mathrm{~nm}$. 
TABLE 2. Growth response of strain $12 \mathrm{MS}^{\mathrm{T}}$ and other mollicutes to cholesterol

\begin{tabular}{|c|c|c|c|c|c|c|}
\hline \multicolumn{4}{|c|}{ Presence in test medium of: } & \multicolumn{3}{|c|}{$\begin{array}{l}\text { Amt of protein } \\
(\mathrm{mg} / \mathrm{ml}) \text { in test } \\
\quad \text { organism }^{a} \text { : }\end{array}$} \\
\hline $\begin{array}{c}\text { Albumin } \\
(1 \%)\end{array}$ & $\begin{array}{l}\text { Palmitic acid } \\
(10 \mu \mathrm{g} / \mathrm{ml})\end{array}$ & $\begin{array}{c}\text { Tween } 80 \\
(0.01 \%)\end{array}$ & $\begin{array}{l}\text { Cholesterol, } \\
\text { at a concn } \\
(\mu \mathrm{g} / \mathrm{ml}) \text { of: }\end{array}$ & $12 \mathrm{MS}^{\mathrm{T}}$ & G122 & PC \\
\hline- & - & - & 10 & 0 & 0 & 4.4 \\
\hline- & - & - & 0 & 0 & 0 & 4.7 \\
\hline+ & + & - & 0 & 0 & 0 & 4.4 \\
\hline+ & + & + & 0 & 0 & 0 & 4. \\
\hline+ & + & + & 1 & 0.9 & 1.0 & 3.9 \\
\hline+ & + & + & 5 & 1.8 & 2.1 & 4.2 \\
\hline+ & + & + & 10 & 3.6 & 2.9 & 4.8 \\
\hline+ & + & + & 20 & 5.0 & 4.8 & 4.0 \\
\hline
\end{tabular}

${ }^{a}$ G122, M. caviae G122 ${ }^{\mathrm{T}}$; PG8, Acholeplasma laidlawii $\mathrm{PG} 8^{\mathrm{T}}$.

Taxonomic assignment. The properties described above for strain $12 \mathrm{MS}^{\mathrm{T}}$ fulfill the criteria (17) for species descriptions of members of the class Mollicutes. A cell wall is absent, and the cells can be filtered through 200- and 450-nm-pore-size membranes, fail to revert to walled bacteria when they are grown in antibiotic-free media, have low $\mathrm{G}+\mathrm{C}$ contents, are resistant to penicillin, and produce typical fried-egg colonies on solid media. The growth requirement for sterol or serum, in conjunction with the lack of helicity, places these organisms in the order Mycoplasmatales of the family Mycoplasmataceae. The inability of the strains to hydrolyze urea mandates assignment to the genus Mycoplasma.

Strain $12 \mathrm{MS}^{\mathrm{T}}$ and the other mycoplasma strains isolated from Afghan pikas belong to the same species, as they have identical biological characteristics and identical serological properties. The lack of serological relatedness of strain $12 \mathrm{MS}^{\mathrm{T}}$ to other Mollicutes species demonstrates that the isolates from Afghan pikas represent a previously unrecognized species, for which the name Mycoplasma lagogenitalium is proposed. The taxonomic description below summarizes the properties of this new species.

Description of Mycoplasma lagogenitalium sp. nov. Mycoplasma lagogenitalium (la. go. ge. ni. ta'. li. um; lagos, Gr. masc. n., hare; genitalium, L. plur. neutr. gen., of hare's genitals). Cells lack true cell walls and are coccoid. Colonies on solid medium usually have a typical fried-egg appearance. Chemoorganotroph. Ferments glucose. Arginine and urea are not hydrolyzed. Reduces tetrazolium chloride. Possesses phosphatase activity. Films or spots are not produced. Lyses sheep erythrocytes but does not adsorb sheep, rabbit, or horse erythrocytes. Cholesterol or serum is required for growth. The growth temperature is $37^{\circ} \mathrm{C}$. The $\mathrm{G}+\mathrm{C}$ content of the DNA is $23 \pm 1.0$ mol\%. Isolated from the preputial smegma of Afghan pikas
(Ochotona rufescens rufescens). Pathogenicity has not been demonstrated. The type strain is $12 \mathrm{MS}$ (= ATCC $700289^{\mathrm{T}}$ ).

\section{ACKNOWLEDGMENTS}

We thank Y. Kosako and Y. Benno, Japan Collection of Microorganisms, the Institute of Physical and Chemical Research, for performing the DNA composition test and all workers who provided mycoplasma strains and antisera listed in Table 1.

\section{REFERENCES}

1. Aluotto, B. B., R. G. Wittler, C. O. Williams, and J. E. Faber. 1970. Standardized bacteriologic techniques for the characterization of Mycoplasma species. Int. J. Syst. Bacteriol. 20:35-58

2. Bradbury, J. M. 1977. Rapid biochemical tests for characterization of $M y-$ coplasmatales. J. Clin. Microbiol. 5:70-76.

3. Cassell, G. H., and A. Hill. 1979. Murine and other small-animal mycoplasmas, p. 235-273. In J. G. Tully and R. F. Whitcomb (ed.), The mycoplasmas, vol. 2. Academic Press, Inc., New York, N.Y.

4. Clyde, W. A., Jr. 1964. Mycoplasma species identification based upon growth inhibition by specific antisera. J. Immunol. 92:958-965.

5. Deeb, B. J., and G. E. Kenny. 1967. Characterization of Mycoplasma pulmonis variants isolated from rabbits. J. Bacteriol. 93:1416-1429.

6. Edward, D. G. 1971. Determination of sterol requirement for Mycoplasmatales. J. Gen. Microbiol. 69:205-210.

7. Edward, D. G., and W. A. Fitzgerald. 1951. Cholesterol in the growth of organisms of the pleuropneumonia group. J. Gen. Microbiol. 5:576-586.

8. Hill, A. C. 1983. Mycoplasma cricetuli, a new species from the conjunctivas of chinese hamsters. Int. J. Syst. Bacteriol. 33:113-117.

9. Hill, A. C. 1983. Mycoplasma collis, a new species isolated from rats and mice. Int. J. Syst. Bacteriol. 33:847-851.

10. Hill, A. C. 1984. Mycoplasma cavipharyngis, a new species from the nasopharynx of guinea pigs. J. Gen. Microbiol. 130:3183-3188.

11. Ishihara, C. 1977. Characterization of mycoplasma isolated from rabbits. Bull. Inst. Immunol. Sci. Hokkaido Univ. 37:29-32.

12. Kotani, H., and G. J. McGarrity. 1985. Rapid and simple identification of mycoplasmas by immunobinding. J. Immunol. Methods 85:257-267.

13. Marmur, J., and P. Doty. 1962. Determination of the base composition of deoxyribonucleic acid from its thermal denaturation temperature. J. Mol. Biol. 5:109-118.

14. McGarrity, G. J., D. L. Rose, V. Kwiatkowski, A. S. Dion, D. M. Phillips, and J. G. Tully. 1983. Mycoplasma muris, a new species from laboratory mice. Int. J. Syst. Bacteriol. 33:350-355.

15. Morton, H. E., and R. J. Roberts. 1967. Production of antimycoplasma (PPLO) antibodies in rabbits. Proc. Soc. Exp. Biol. Med. 125:538-543.

16. Sobeslavsky, O., B. Prescott, and R. M. Chanock. 1968. Absorption of Mycoplasma pneumoniae to neuraminic acid receptors of various cells and possible role in virulence. J. Bacteriol. 96:695-705.

17. Subcommittee on the Taxonomy of Mollicutes of the International Committee on Systematic Bacteriology. 1995. Revised minimum standards for description of new species of the class Mollicutes (division Tenericutes). Int. J. Syst. Bacteriol. 45:605-612.

18. Taylor-Robinson, D., R. H. Purcell, D. C. Wong, and R. M. Chanock. 1966. A color test for the measurement of antibody to certain mycoplasma species based upon the inhibition of acid production. J. Hyg. 64:91-104.

19. Tully, J. G. 1983. Cloning and filtration techniques for mycoplasmas. Methods Mycoplasmol. 1:355-362.

20. Venable, J. H., and R. Coggeshall. 1965. A simplified lead citrate stain for use in electron microscopy. J. Cell Biol. 25:407.

21. Williams, C. O., and R. G. Wittler. 1971. Hydrolysis of esculin and phosphatase production by members of the order Mycoplasmatales which do not require sterol. Int. J. Syst. Bacteriol. 21:73-77. 